\title{
Crianças e adolescentes com problemas emocionais e comportamentais têm necessidade de políticas de inclusão escolar?
}

\section{Do children and adolescents with emotional and behavioral problems need educational inclusion policies?}

\author{
Fabiana Vieira Gauy ${ }^{1}$
}

\begin{abstract}
RESUMO
Este trabalho teve o objetivo de avaliar o perfil de crianças encaminhadas para serviços de saúde mental e discutir o impacto dos problemas funcionais apresentados em escolares, que podem estar sendo negligenciados pelas políticas públicas brasileiras. Os dados de 160 escolares, de ambos os sexos, foram coletados em três serviços médicos de saúde mental com os respectivos cuidadores, a partir de dois instrumentos: ficha de identificação para caracterizar os participantes, os cuidadores, o perfil sociodemográfico, o responsável pelo encaminhamento e a queixa; e Inventário dos Comportamentos para Crianças e Adolescentes entre 6 e 18 anos (CBCL6/18 anos). Os dados obtidos evidenciam uma alta demanda devido a dificuldades emocionais/comportamentais, combinadas com dificuldades de aprendizagem, de relacionamento e na vida diária, denominadas pelo Ministério da Educação (MEC) como transtornos funcionais, não atendidas pelas políticas de Ensino Especial, encaminhadas por postos de saúde e médicos, e não prioritariamente pela escola, encaminhador padrão. Exceto pelo encaminhamento, os dados são consistentes com a literatura, que aponta uma alta incidência dessas dificuldades em crianças e adolescentes, geradores de barreiras acadêmicas e de problemas de alto impacto ao longo da vida acadêmica e pessoal. A esse respeito, modelos de intervenções baseadas em escola têm de forma crescente ampliado o conceito de inclusão e apontado alternativas
\end{abstract}

DOI: $10.1590 / 0104-4060.44687$

1 Universidade de Brasília, Instituto de Psicologia. Brasília, Distrito Federal, Brasil. SQN 212 Bloco K apto 606 Asa Norte. CEP: 70864-110.E-mail: fabianagauy@gmail.com 
promissoras de aliança entre escola e serviços de saúde mental, o que pode vir a inspirar futuras políticas públicas na área.

Palavras-chave: problemas emocionais; problemas comportamentais; serviços de saúde mental; infância e adolescência; políticas educacionais de inclusão.

\begin{abstract}
This study had the objective of evaluating the profile of children referred to mental health services and of discussing the impact of functional problems presented at school, which may be neglected by the Brazilian public policies. Data from 160 schoolchildren, of both genders, was collected from their respective caretakers in three mental health services using two instruments: an identification form to characterize the participants, the caretakers, the social-demographic profile, the person responsible for the referral and demand; and the Child Behavior Checklist (CBCL) for children and adolescents between 6 and 18 years of age. Data obtained showed a high demand due to emotional/behavioral difficulties combined with learning, relationship and daily life difficulties named functional disorders by the Brazilian Ministry of Education and Culture (MEC) and not attended by the special education policies, which were referred by health centers and not mainly by the school, which would be the standard. Except for the referral, data is consistent with the literature, which points out a high incidence of these difficulties in children and adolescents, creating academic barriers and problems that have a great impact throughout their academic and personal lives. In this regard, intervention models based in schools have increasingly widened the concept of inclusion and offered promising alternatives of alliances between schools and mental health services, which may inspire future public policies in the area.
\end{abstract}

Keywords: emotional problems; behavioral problems; mental health services; childhood and adolescence; educational inclusion policies.

As ações e programas da extinta Secretaria de Educação Especial (Seesp), do Ministério da Educação, atualmente fazem parte das atribuições da Secretaria de Educação Continuada, Alfabetização, Diversidade e Inclusão (SECADI). Segundo o Decreto $\mathrm{n}^{\circ} 7.611$, de 17 de novembro de 2011, cabe à União incentivar a oferta ao atendimento educacional especializado apenas aos alunos com deficiência físicas, motoras e intelectuais; com transtornos globais do desenvolvimento; e com altas habilidades ou superdotação, matriculados na rede pública de ensino regular, a partir da capacitação de gestores e professores, 
do oferecimento de estrutura física, de apoio técnico e financeiro e da produção e distribuição de recursos educacionais, que proporcionem mobilidade e acessibilidade. Considera-se, nesse decreto, que "[...] atendimento educacional especializado é o conjunto de atividades, recursos de acessibilidade e pedagógicos organizados institucionalmente, prestado de forma complementar ou suplementar à formação dos alunos no ensino regular"; e que este atendimento deve fazer parte da proposta pedagógica, envolver a família e se articular com as demais políticas públicas. (BRASIL, 2011).

Anualmente, o Instituto Nacional de Estudos e Pesquisas Educacionais Anísio Teixeira (INEP) (<www.inep.gov.br $>$ ) realiza um levantamento estatístico sobre a educação básica no País, que permite tanto acompanhar o impacto das políticas educacionais adotadas, como também subsidiar a formulação e a implementação de políticas públicas. Os dados finais do Censo Escolar de 2014 contabilizaram 49.771.371 matrículas e identificaram que em torno de $3,5 \%$ dessas se referiam a alunos portadores de necessidades educativas especiais, matriculados em classe comum. Não se qualificam como portadores de necessidades especiais aqueles com transtornos funcionais, como Transtorno de Déficit de Atenção, Dislexia, Transtorno de Ansiedade.

\section{Transtornos funcionais em escolares}

Kendall e Comer (2010) enfatizam os quadros patológicos mais comuns, considerados pelo MEC como transtornos funcionais, agrupando-os em sete categorias principais, a saber: (a) transtornos de comportamento disruptivo, (b) transtornos emocionais; (c) transtornos alimentares; (d) retardo mental; (e) transtornos de aprendizagem; (f) transtornos invasivos do desenvolvimento; e (g) transtorno de excreção. Entre essas categorias, destacam-se as duas primeiras como as de maior incidência em escolares, embora seja necessário ressaltar que as dificuldades mencionadas geralmente se apresentam de forma combinada entre si, e não isolada. Nota-se, ainda, que somente dois dos referidos transtornos são incluídos na política de inclusão - retardo mental e transtornos globais do desenvolvimento.

As dificuldades associadas aos transtornos de comportamento disruptivo, ou problemas externalizantes, são aquelas associadas a comportamentos manifestos, como comportamento agressivo a outros ou a propriedades, comportamento rebelde, cruel, desobediente a regras e a autoridades, ou comportamento de desatenção e agitação. Estima-se que em torno de 4 a 5\% da população es- 
colar apresente pelo menos uma dessas dificuldades, sendo mais comum, como mencionado, a apresentação combinada entre elas. (ROHDE et al., 2000). Esses problemas são bastante associados à baixa tolerância à frustração e à rejeição dos pares escolares, assim como são mais comuns em meninos com idade entre 4 e 16 anos. O curso do transtorno tende a ser mais crônico e o prognóstico pior quando os sintomas surgem na infância do que quando surgem na adolescência, quando podem ser isolados ou transitórios. (KENDALL; COMER, 2010). Por perturbarem o ambiente da sala de aula, tendem a ser mais rapidamente identificados pelos outros alunos e professores e constituem o foco do maior número de programas de intervenção citados na literatura. (CHRISTENSON; HAVSY, 2004; VAN ARCKER, 2007; WEBSTER-STRATTON; REID, 2003; WEISZ; HAWLEY, 2002). Aqui se incluem os transtornos de conduta e déficit de atenção/hiperatividade. É alta a associação ou comorbidade entre transtornos disruptivos e transtornos de aprendizagem, que se referem a desempenho escolar substancialmente abaixo do esperado pela idade, inteligência e educação (KENDALL; COMER, 2010; ROHDE et al., 2000), que compromete diferentes esferas acadêmicas. A partir da nova classificação americana, não há mais subdivisões nessa categoria, como transtorno de leitura, transtorno de cálculo, entre outras. (AMERICAN PSYCHIATRIC ASSOCIATION, 2014).

Os transtornos emocionais, ou problemas internalizantes, são aqueles associados a dificuldades emocionais, como ansiedade patológica e depressão. Por não perturbarem o ambiente escolar, são pouco reconhecidos naquele âmbito. A ansiedade patológica é caracterizada por medo patológico e preocupação desproporcional com relação a um determinado estímulo. Geralmente crianças e jovens apresentam, além dos medos e preocupações irracionais, queixas somáticas - principalmente dores de cabeça e dores abdominais, e dificuldades para relaxar, sendo mais comum em meninas. (CASTILLO et al., 2000; KENDALL; COMER, 2010). Por sua vez, os transtornos depressivos são caracterizados por tristeza ou irritabilidade intensa, não justificadas por fatores estressores situacionais. Queixas comuns incluem: cansaço fácil, isolamento, falta de prazer e queixas somáticas inespecíficas, sendo as dores de cabeças e dores na barriga as mais comuns. A criança pode passar a ter comportamentos de irritabilidade e agressividade, choro, pesadelos e queixas de cansaço. O adolescente pode apresentar hipersonia, melancolia, perda de peso, abuso de substância lícita ou ilícita e tentativas de suicídio. Aproximadamente 43\% dos casos apresentam comorbidade com transtornos ansiosos e transtornos disruptivos. Aproximadamente $21 \%$ dos adolescentes deprimidos evoluem para transtorno bipolar, que leva a flutuações de humor que podem variar de euforia/irritabilidade a depressão. (FU I; CURATOLO; FRIEDRICH, 2000; WEISZ; HAWLEY, 2002). Inclui-se aqui o transtorno de ansiedade social, as fobias, o transtorno de ansiedade 
generalizada, o mutismo seletivo, a ansiedade de separação e o transtorno do pânico, como transtornos de ansiedade; e transtorno depressivo e depressivo persistente, como transtornos depressivos. Na atual versão da classificação de transtornos mentais americana foram caracterizados dois novos transtornos depressivos, a saber: Transtorno Disruptivo de Desregulação do Humor, caracterizado por temperamento explosivo desproporcional, identificado entre 6 e 18 anos, e o Transtorno Disfórico Pré-mestrual, caracterizado por flutuações de humor associadas a alterações hormonais em jovens e mulheres em idade fértil. (AMERICAN PSYCHIATRIC ASSOCIATION, 2014).

Os transtornos alimentares em escolares são associados à ingestão persistente de substâncias que não são nutritivas, nem adequadas para alimentação, como ingestão de cabelo ou de terra (pica); a repetida regurgitação e remastigação do alimento, sem causa médica (ruminação); ou a própria alimentação, com restrição alimentar pela preocupação excessiva com o peso (anorexia/bulimia). Enquanto ingestão de substâncias impróprias é comum em crianças pequenas ou com retardo mental, sobretudo se há histórico na família, a anorexia e a bulimia já são mais comuns no início da adolescência. (AMERICAN PSYCHIATRIC ASSOCIATION, 2014). Para anorexia e bulimia, a dieta é considerada o gatilho ou desencadeador mais comum. (GONÇALVEZ et al., 2013; KENDALL; COMER, 2010).

Os transtornos invasivos do desenvolvimento são dificuldades associadas a prejuízo na comunicação, interação social e padrões estereotipados de comportamento. Escolares com transtornos invasivos costumam também apresentar retardo mental, que leva à limitação substancial do funcionamento intelectual e limitações adaptativas de habilidades da vida diária. $\mathrm{Na}$ nova classificação americana incluiu-se aqui o Autismo, Asperger, Transtorno Desintegrativo da Infância e Transtornos Invasivos do Desenvolvimento Sem outras Especificações. (AMERICAN PSYCHIATRIC ASSOCIATION, 2014). Ressalta-se, a esse respeito, o fenômeno atual de um número crescente de casos de autismo diagnosticados em escolares.

E, por fim, os transtornos de excreção, associados à evacuação repetida em lugares inadequados, como na cama ou na roupa. Diferenciados por encoprese - evacuação inadequada de fezes, que pode ser retentiva (prisão de ventre) ou não retentiva (escapes de fezes nas roupas seria um exemplo) (COELHO, 2001); ou enurese - evacuação de urina, que pode ser diurna (micção repetida em roupas) ou noturna (micção na cama no período do sono). A literatura aponta que essas dificuldades, em especial a enurese, são geradoras de problemas emocionais, como a timidez e o retraimento social. (ARANTES; SILVARES, 2007). Tanto a encoprese como a enurese são classificadas como primárias, quando nunca houve controle anal ou vesical; ou secundárias, quando houve 
controle em algum momento, mas devido a algum fator a criança voltou a ter escapes. (KENDALL; COMER, 2010).

Essas dificuldades funcionais supracitadas: (a) levam a barreiras acadêmicas, de impacto na escola e no curso do desenvolvimento normal (COSTELLO; FOLEY; ANGOLD, 2006; GAUY; ROCHA, 2014; KENDALL; COMER, 2010; PATEL et al., 2007); (b) são de alta incidência - em torno de $10 \%$ a $20 \%$ da população escolar matriculada (COSTELLO; FOLEY; ANGOLD, 2006); e (c) exigem capacitação dos gestores/professores, estrutura física, de apoio técnico e financeiro, e da produção e distribuição de recursos educacionais. (PATEL et al., 2007). Isso posto, o objetivo deste artigo foi fazer um levantamento de problemas em escolares encaminhados para serviços de saúde mental e discutir sobre intervenções baseadas na escola, com especial atenção para os estudos que incidem sobre a saúde mental infanto-juvenil.

\section{Método}

\section{Participantes}

Participaram deste estudo 160 escolares de ambos os sexos, sendo que a maioria tinha idade entre 10 e 14 anos $(51,9 \%)$, era do sexo masculino $(63,1 \%)$, cursava Ensino Fundamental (80,5\%), nenhum era de Ensino Especial, morava com os pais biológicos (85\%), com idade entre 35 e 44 anos (42,5\%), com Ensino Médio (28,1\%), em união estável - família biparental (61,4\%), trabalhadores de serviços e do comércio $(25,8 \%)$, com renda familiar de 2 a 4 salários mínimos $(53,8 \%)$ e pertencentes à classe social " $\mathrm{C}$ " $(63 \%)$, segundo as normas Critério Brasil do Instituto Brasileiro de Geografia e Estatística (IBGE).

\section{Instrumentos}

Ficha de Identificação, dividida em duas partes: a primeira, com os dados de identificação do usuário do serviço (nome, idade, escolaridade, endereço, telefone e queixa) e do cuidador (nome, escolaridade, estado civil e relação com o usuário); e a segunda parte, com os dados socioeconômicos avaliados pelo Critério Brasil do IBGE.

Inventário dos Comportamentos para Crianças e Adolescentes entre 6 a 18 anos (CBCL6/18 anos), que avalia a percepção parental sobre o filho em 138 itens (ROCHA et al., 2013). As respostas dos pais são analisadas por um software que transforma os escores brutos em "Escores T" - medida estatística 
utilizada pelo referido sistema, que converte diferentes medidas em escalas individuais e soma de escalas de competências e de problemas. As Escalas Individuais de Competência se diferenciam em três: Competência em Atividade, Competência Social e Competência Escolar. As Escalas de Problemas se diferenciam em oito escalas individuais e três somas de escalas: (a) Ansiedade/ Depressão, Retraimento/Depressão, Queixas Somáticas - que juntas configuram a Escala de Internalização; (b) Problemas de Sociabilidade, Problemas com Pensamento, Problemas de Atenção; (c) Violação de Quebra de Regras e Comportamento Agressivo, que juntas configuram a Escala de Externalização. As somas de todas as escalas individuais de problemas configuram a Escala Total de Problemas. O software que analisa os dados diferencia os escores T em perfis não clínico, limítrofe e clínico. Para este estudo, os escores limítrofes e clínicos foram agrupados como clínicos, ou seja, com índices maiores que o esperado considerando idade e gênero.

\section{Procedimento}

Os dados foram coletados considerando a rotina dos serviços médicos de saúde mental de duas regiões brasileiras, Centro-Oeste e Sudeste, de modo a interferir o menos possível no andamento dos atendimentos. Os instrumentos foram aplicados nos pais dos escolares, que eram abordados por um dos seis auxiliares de pesquisa, alunos do curso de Psicologia, enquanto esperavam o atendimento do filho. Aos pais e aos filhos foi explicado o objetivo do estudo e entregue o termo de consentimento para uso dos dados. Todos os abordados, durante o mês que durou o estudo, aceitaram participar. Após assinarem o termo de consentimento, iniciava-se a aplicação dos instrumentos. Nesse momento, era perguntando se eles preferiam que os instrumentos fossem lidos em voz alta, com a resposta registrada pelo auxiliar de pesquisa, ou se preferiam responder sozinhos ao inventário.

Para aqueles que pediam para ler em voz alta, era entregue outra cópia do instrumento para que pudessem acompanhar a leitura. Nas duas condições de registro, o auxiliar de pesquisa mantinha-se ao lado do cuidador para sanar qualquer dúvida. Na Ficha de Identificação o dado poderia ser fornecido pelo cuidador ou pelo filho. Já no CBCL, o dado coletado era apenas o relatado pelo cuidador. O tempo médio gasto para a aplicação dos instrumentos foi de uma hora e trinta minutos.

Os dados foram registrados em uma planilha considerando porcentagem e frequência. Na análise dos dados relacionados às queixas obtidas no $\mathrm{CBCL}$, considerou-se a distribuição dos escores clínicos e não clínicos fornecida pelo software. 


\section{Resultados e Discussão}

Os resultados aqui apresentados se referem aos dados de encaminhamento e queixa, coletados na Ficha de Identificação, e os dados de Competências e Problemas, coletados pelo $\boldsymbol{C B C L}$, e focaram em responder a duas interrogativas, a saber: Quem encaminhou para o serviço?; e Quais foram os problemas coletados pelos instrumentos?

\section{Quem encaminhou para os serviços de saúde mental?}

A Tabela 1 dispõe as variáveis de encaminhamentos para os serviços de saúde mental.

TABELA 1 - DISTRIBUIÇÃO DO ENCAMINHAMENTO PARA OS SERVIÇOS DE SAÚDE MENTAL, EM PORCENTAGEM E FREQUÊNCIA

\begin{tabular}{ll}
\hline Encaminhamento & $\mathbf{\%}(\mathbf{n})$ \\
\hline Serviços de Psicologia & $8,1 \%(13)$ \\
Serviço Hospitalar & $12,5 \%(20)$ \\
Posto de Saúde & $11,9 \%(19)$ \\
Médicos & \\
$\quad$ Clínico geral & $2,5 \%(4)$ \\
$\quad$ Psiquiatra & $10,6 \%(17)$ \\
$\quad$ Neurologista & $4,4 \%(7)$ \\
Fonoaudióloga & $0,6 \%(1)$ \\
Escola & $5,0 \%(8)$ \\
Espontâneo & $6,3 \%(10)$ \\
Família & $2,5 \%(4)$ \\
Conhecido & $6,9 \%(11)$ \\
Outro paciente & $1,3 \%(2)$ \\
Mídia & $13,1 \%(21)$ \\
Outros & $3,1 \%(5)$ \\
Não consta & $11,3 \%(18)$ \\
\hline
\end{tabular}

FONTE: A autora (2015).

Em relação aos dados apresentados na Tabela 1, três pontos chamam atenção: (a) os principais encaminhadores para os serviços estudados foram os serviços de saúde (hospital e posto de saúde) ou profissionais de saúde (médicos) (41,9\%). Esse dado está consonante a outros estudos que têm demonstrado o aumento do encaminhamento por parte de outros serviços de saúde 
(ver DELVAN et al., 2010; LOUZADA, 2003; MELO; PERFEITO, 2006), e sugere que os profissionais de saúde estão mais capacitados do que outrora para encaminhar para os serviços especializados em saúde mental; (b) o impacto da mídia no esclarecimento dos procedimentos e serviços de saúde mental motivou a busca pelos serviços $(13,1 \%)$, e provavelmente tenha favorecido na busca espontânea de $6,3 \%$ dos usuários do serviço, indicando uma diminuição do estigma da busca para esses serviços, tão significativo em outros tempos; e (c) o reduzido encaminhamento por parte das escolas $(5 \%)$, encaminhador típico da clientela em idade escolar nos último 30 anos. (ANCONA-LOPEZ, 1983; MELO; PERFEITO, 2006; SILVARES, 1998).

Ao longo dos últimos 50 anos, houve uma crescente preocupação em encontrar as melhores formas de promover o desenvolvimento saudável, reduzir o risco e melhorar os problemas de saúde mental de escolares, principalmente os transtornos disruptivos, emocionais e os invasivos do desenvolvimento, em todo o mundo ocidental. Nesse período, a qualidade e a eficácia de tratamentos e dos serviços de saúde mental, a capacitação dos profissionais de saúde e a divulgação dos problemas de saúde mental em todas as mídias cresceram significativamente. Contudo, a adesão aos tratamentos ainda é baixa, o que compromete o resultado terapêutico e a manuteção da melhora do quadro clínico, de maneira que a aliança entre os serviços de saúde mental e a educação parece ser uma boa saída.

Por isso, é preocupante o fato de a avaliação do impacto das intervenções de saúde mental no ambiente acadêmico ser escassa e pouco comentada no Brasil, que ainda avalia os escolares especiais de forma muito restrita, considerando os transtornos funcionais como um problema da saúde ou um problema pessoal e não da educação. Ainda mais preocupante, em que pese o tamanho reduzido da amostra deste estudo, é o reduzido encaminhamento por parte da escola. Esse resultado frustra a expectativa de que a escola tenha boa sensibilidade para identificar os problemas funcionais, inclusive antes mesmo do que os pais, devido à experiência e ao conhecimento acumulados sobre o desenvolvimento infanto-juvenil.

\section{Quais foram os problemas coletados pelos instrumentos?}

Os dados sobre as queixas motivadoras pela busca do atendimento, coletados na ficha de identificação com os cuidadores e filhos e os dados coletados pelo CBCL são apresentados, respectivamente, nas Tabelas 2 e 3. 
TABELA 2 - DISTRIBUIÇÃO DOS PROBLEMAS QUE MOTIVARAM A BUSCA PELOS SERVIÇOS DE SAÚDE MENTAL, EM PORCENTAGEM E FREQUÊNCIA

\begin{tabular}{ll}
\hline Queixa & \% (n) \\
\hline Dificuldades de aprendizagem & $53,5 \%(85)$ \\
Dificuldades emocionais e comportamentais & $81,8 \%(130)$ \\
Dificuldades de relacionamento interpessoal & $32,7 \%(52)$ \\
Dificuldades da vida diária & $32,1 \%(51)$ \\
Dificuldades da esfera sexual & $5,7 \%(9)$ \\
Sintomas físicos & $17,0 \%(27)$ \\
Distúrbios orgânicos & $15,1 \%(24)$ \\
Distúrbios da alimentação e sono & $27,7 \%(44)$ \\
Dependência química & $3,8 \%(6)$ \\
Distúrbios psiquiátricos & $26,4 \%(42)$ \\
\hline
\end{tabular}

FONTE: A autora (2015).

$\mathrm{Na}$ Tabela 2, observa-se que a queixa Dificuldades de aprendizagem $(53,5 \%)$, descrita pelo instrumento como dificuldades para aprender, incluindo leitura, escrita e interpretação, foi a segunda mais citada, atrás apenas da queixa Dificuldades emocionais e comportamentais $(81,8 \%)$, descrita como agressividade, ansiedade, isolamento social, depressão, choro frequente, dependência, imaturidade, medo, agressividade e violação de regras. A terceira queixa mais identificada foi Dificuldades de relacionamento interpessoal $(32,7 \%)$, descrita como dificuldade de fazer e manter amigos, de solução de problemas interpessoais, empatia e assertividade; seguida por Dificuldades da vida diária $(32,1 \%)$, descrita por dependência de outra pessoa para executar tarefas que já seriam esperadas de serem realizadas sem ajuda, de acordo com a faixa etária, e baixa capacidade de resolver os próprios problemas sozinho. Os cuidadores relataram pelo menos 2 dificuldades-queixa por filho. Essa combinação entre dificuldades vai ao encontro de estudos recentes, que indicam a alta incidência de comorbidades na população infanto-juvenil (COSTELLO; FOLEY; ANGOLD, 2006; DELVAN et al., 2010; LOUZADA, 2003; MELO; PERFEITO, 2006), sugerindo a necessidade de ações intersetoriais entre atenção básica, educação e saúde mental para que os tratamentos sejam mais eficazes. (TANAKA; LAURIDSEN-RIBEIRO, 2009; TRESCO; LEFLER; POWER, 2010).

Os dados coletados pelo CBCL foram analisados segundo as Escalas Individuais e as Soma das Escalas, para Competência e Problemas, conforme o agrupamento do software (Tabela 3). 
TABELA 3 - DISTRIBUIÇÃO DOS PROBLEMAS COLETADOS NO CBCL, EM PORCENTAGEM E FREQUÊNCIA

\begin{tabular}{|c|c|c|c|c|}
\hline & Escalas Individuais & $\%(n)$ & Soma das Escalas & $\%(n)$ \\
\hline \multirow{9}{*}{ 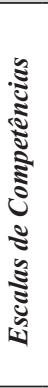 } & $\begin{array}{l}\text { Competência em Atividades } \\
(n=157)\end{array}$ & & $\begin{array}{l}\text { Soma de Competência } \\
(n=146)\end{array}$ & \\
\hline & Não clínico & $44,6 \%(70)$ & Não clínico & $15,1 \%(22)$ \\
\hline & Clínico & $55,4 \%(87)$ & Clínico & $84,9 \%(124)$ \\
\hline & Competência Social $(n=159)$ & & & \\
\hline & Não clínico & $60,4 \%(96)$ & & \\
\hline & Clínico & $39,6 \%(63)$ & & \\
\hline & Competência Escolar $(n=149)$ & & & \\
\hline & Não clínico & $59,1 \%(88)$ & & \\
\hline & Clínico & $40,9 \%(61)$ & & \\
\hline \multirow{24}{*}{ 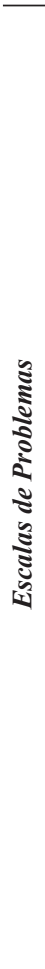 } & Ansiedade/Depressão & & Escala de Internalização & \\
\hline & Não clínico & $25,6 \%(41)$ & Não clínico & $10 \%(16)$ \\
\hline & Clínico & $74,4 \%(119)$ & Clínico & $90 \%(144)$ \\
\hline & Retraimento/Depressão & & Escala de Externalização & \\
\hline & Não clínico & $30,6 \%(49)$ & Não clínico & $30,6 \%(49)$ \\
\hline & Clínico & $69,4 \%(111)$ & Clínico & $69,4 \%(111)$ \\
\hline & Queixas Somáticas & & Escala Total de Problemas & \\
\hline & Não clínico & $53,1 \%(85)$ & Não clínico & $14,4 \%(23)$ \\
\hline & Clínico & $46,9 \%(75)$ & Clínico & $85,6 \%(137)$ \\
\hline & Problemas de Sociabilidade & & & \\
\hline & Não clínico & $43,8 \%(70)$ & & \\
\hline & Clínico & $56,3 \%(90)$ & & \\
\hline & $\begin{array}{l}\text { Problemas com o } \\
\text { Pensamento }\end{array}$ & & & \\
\hline & Não clínico & $41,9 \%(67)$ & & \\
\hline & Clínico & $58,1 \%(93)$ & & \\
\hline & Problemas de Atenção & & & \\
\hline & Não clínico & $45 \%(72)$ & & \\
\hline & Clínico & $55 \%(88)$ & & \\
\hline & Violação de Regras & & & \\
\hline & Não clínico & $69,4 \%(111)$ & & \\
\hline & Clínico & $30,6 \%(49)$ & & \\
\hline & Comportamento Agressivo & & & \\
\hline & Não clínico & $41,9 \%(67)$ & & \\
\hline & Clínico & $58,1 \%(93)$ & & \\
\hline
\end{tabular}

FONTE: A autora (2015).

Na Tabela 3, segundo os cuidadores, a maioria dos filhos atingiu perfil clínico em 11 das 15 escalas do CBCL analisadas, sendo que os índices clíni- 
cos foram mais frequentes em Problemas de Internalização (90\%), Total de Problemas (85,6\%), Soma de Competências (84,9\%) e na Escala de Ansiedade/Depressão (74,4\%). Nesses dados também se observa uma contradição. Apesar de os cuidadores identificarem como frequentes as dificuldades de aprendizagem - em 53,5\% dos casos na Ficha de Identificação -, no CBCL apenas $40,9 \%$ avaliaram o filho com problemas de competência escolar. Aventa-se a possibilidade de que o baixo encaminhamento da escola para os serviços de saúde mental pode ter sido influenciado pela combinação de dificuldades predominantemente emocionais, representadas por retraimento, ansiedade, depressão e queixas somáticas, dificuldades que perturbam menos escolar, do que os problemas externalizantes.

De todo modo, há um amplo entendimento, a partir dos estudos na área de saúde mental, de que a falta de programas de atendimento e prevenção em problemas de saúde mental infanto-juvenil, bem como a ausência de políticas que mapeiem e capacitem os profissionais, contribuem para predizer um desenvolvimento futuro devastador, principalmente no que diz respeito à população aqui estudada. Dazzani et al. (2014) ainda alertam, a partir de uma revisão de 21 estudos sobre "queixa escolar", que, além da falta de políticas públicas e capacitação, há uma tendência por parte dos profissionais da psicologia e da educação de responsabilização exclusiva de pais e alunos pelo fracasso escolar, e, por conseguinte, uma postura de exclusão desses alunos por parte da escola, favorecendo a patologização do escolar e sua correspondente medicalização. Os recentes achados demonstram de forma consistente a necessidade de maior investimento, para que se possa articular uma rede de atendimento com capacidade técnica para absorver a demanda de forma resolutiva (BELFER, 2008; COUTO; DUARTE; DELGADO, 2008) e construir um sistema mais acolhedor, menos opressor e excludente.

O estudo de Christenson e Havsy (2004) examinou os resultados de intervenções aplicadas em escolas a partir de uma revisão de 24 trabalhos. Os dados apontam uma maioria de intervenções voltadas para a Educação Infantil e Ensino Fundamental, com um enfoque predominante sobre a prevenção de comportamentos antissociais. Os programas que alcançaram maior impacto positivo eram aplicados de forma intensiva e envolviam vários componentes, como professores, pais e alunos, e metas, como, por exemplo, diminuição dos comportamentos-problema e melhoria do desempenho acadêmico.

As intervenções em escola com foco em competência social também apontam para um maior sucesso nas intervenções intensivas, constantes e que envolvam pais, escola e alunos. As revisões de Zins et al. (2004) e de Welsh et al. (2001) demonstram uma relação recíproca entre competências socioemocionais centradas na pessoa, incluindo a autoconsciência, a consciência social, a 
tomada de decisão responsável, a autogestão e o relacionamento interpessoal; e as competências acadêmicas. Apesar de, tradicionalmente, os programas de intervenção baseados na escola focalizarem o comportamento perturbador em sala de aula, há cada vez mais interesse dessa área na prevenção de problemas diversos mediante a promoção de estratégias instrucionais positivas e da modificação de variáveis contextuais, a serem aplicadas em sala de aula por professores, como regras da sala de aula, gerenciamento e transições de atividades, uso eficiente do tempo, e comunicação efetiva e positiva entre professor e alunos, resolução de problemas, e relacionamento interpessoal, para minimizar não apenas os comportamentos agressivos, como também aqueles associados à ansiedade, depressão e retraimento social.

\section{Conclusão}

Este trabalho teve o objetivo de avaliar o perfil de crianças encaminhadas para serviços de saúde mental e discutir o impacto desses problemas funcionais em escolares, que podem estar sendo negligenciados pelas políticas públicas brasileiras. Os dados obtidos apontam uma alta demanda devido a dificuldades emocionais/comportamentais, combinadas com dificuldades de aprendizagem e de vida diária, encaminhada para os serviços de saúde mental por posto de saúde e médicos, e não pela escola, encaminhador padrão, conforme a literatura. Os dados obtidos junto aos cuidadores pelo CBCL confirmam as dificuldades emocionais ou de internalização como mais frequentes na população estudada, em comparação com as dificuldades comportamentais ou de externalização. Os dados são consistentes com a literatura, que apontam uma alta incidência dessas dificuldades em crianças e adolescentes, com potencial para gerar fracasso acadêmico, prejuízo social, dificuldades de relacionamento, suicídio e abuso de drogas (CASTILLO et al., 2000; COSTELLO; FOLEY; ANGOLD, 2006), o que possivelmente explique em parte por que houve tão pouco encaminhamento das escolas: sabe-se que os professores encaminham para os serviços de saúde mental mais casos associados à violência, agressividade e desobediência às regras escolares e sociais. (ANCONA-LOPEZ, 1983; BROWN et al., 2005; MELO; PERFEITO, 2006; SILVARES, 1998).

Lopes (2008), em uma análise de dados de 371 encaminhamentos de escolares para serviços de psicologia devido a dificuldades pedagógicas e 105 entrevistas com professores do Rio Grande do Sul, evidenciou a diferenciação dos alunos com demanda escolar especial entre "alunos-problema", que apre- 
sentam dificuldades funcionais, segundo o MEC; e alunos com deficiência, que apresentam deficiência sensorial, intelectual ou física. Nesse trabalho, ambos os grupos de alunos são classificados pelos professores como alunos de inclusão. Nas entrevistas com os professores, o estudo indicou, inclusive, que os docentes relatam mais dificuldades de manejo com os "alunos-problema", demonstrando falta de recursos técnicos e estruturais. Seguindo esse raciocínio dos professores, conforme demonstrado pelo trabalho de Lopes (2008), programas de saúde mental atuais têm sido indicados para aplicação nas escolas, com uma prosposta que vai além da inclusão, e sugerem uma sobreposição de procedimentos que fogem do modelo tradicional, como programas aplicados de forma intensiva no período de férias acadêmicas, uso de treinamento de habilidades por vinhetas filmadas ou, ainda, as intervenções aplicadas na escola ao longo do período acadêmico. (CHRISTENSON; HAVSY, 2004; WEBSTER-STRATTON; REID, 2003; WELSH et al., 2001; ZINS et al., 2004). Isso é especialmente encorajador, dada à alta prevalência de transtornos psiquiátricos em crianças e adolescentes, seja de internalização ou de externalização.

As intervenções projetadas para serem aplicadas na escola, focalizando a remoção das barreiras de aprendizagem que podem inclusive levar à evasão escolar - pela falta de interesse no contéudo acadêmico e pelas suspensões/ expulsões repetidas nos casos de externalização -, e/ou o abandono escolar pelas dificuldades emocionais nos casos de internalização -, tendem a ser mais efetivas quando há uma sobreposição de treinamento de gestores e professores, e de aplicação do conteúdo treinado na sala de aula, ano a ano, de forma contínua.

Devido à alta incidência dessas dificuldades na população infanto-juvenil, entende-se que a população aqui estudada também necessita de atendimento educacional especializado, por: (a) exigir capacitação dos profissionais da área de educação em saúde mental; (b) necessitar de recursos pedagógicos específicos, que fujam do modelo acadêmico tradicional, incluindo atividades extraclasse e material mais lúdico; e (c) exigir mudanças estruturais e organizacionais, como salas de aulas menores e com menos alunos, comunicação assertiva entre gestores/professores/alunos/família, e antecipação na resolução de problemas típicos - como adaptação no retorno às atividades acadêmicas após as férias escolares, comportamento mais agitado após o recreio, preparação para as atividades que fogem da rotina escolar. E que esse tipo de atendimento "[...] deve fazer parte da proposta pedagógica, envolver a família e articular com as demais políticas públicas", conforme os termos do Decreto 7.611/2011, que dispõe sobre Educação Especial. 


\section{REFERÊNCIAS}

AMERICAN PSYCHIATRIC ASSOCIATION. Manual Diagnóstico e Estatístico de Transtornos Mentais. Porto Alegre: Artmed, 2014.

ANCONA-LOPES, M. Considerações sobre o atendimento fornecido por clínicas-escola de psicologia. Arquivos Brasileiros de Psicologia, v. 35, n. 2, p. 123-35, 1983.

ARANTES, M. C.; SILVARES, E. F. M. Uma comparação entre crianças e adolescentes com enurese noturna primária: impacto e problemas de comportamento. Estudos de Psicologia, Campinas, v. 24, n. 2, p. 155-160, 2007.

BELFER, M. L. Child and adolescent mental disorders: the magnitude of the problem across the globe. Journal of Child Psychology and Psychiatry, v. 49, n. 3, p. 226-236, 2008.

BRASIL. Decreto $n^{\circ} 7.611$, de 17 de novembro de 2011, que dispõe sobre o ensino especial. 2011. Disponível em: <http://www.planalto.gov.br/ccivil_03/_ato2011-2014/2011/ decreto/d7611.htm>. Acesso em: 03 jan. 2016.

BROWN, R. T.; AMLER, R. W.; FREEMAN, W. S.; PERRIN, J. M.; STEIN, M.T.; FELDMAN, H. M.; PIERCE, K.; WOLRAICH, M. L. Treatment of attention-deficit/ hyperactivity disorder: overview of the evidence. Pediatrics, v. 115, n. 6, p. 749-57, Jun. 2005.

CASTILLO, A. G. L.; RECONDO, R.; ASBAHR, F. R.; MANFRO, G. G. Transtornos de ansiedade. Revista Brasileira de Psiquiatria, São Paulo, v. 22, supl. 2, p. 20-23, dez. 2000.

CHRISTENSON, S. L.; HAVSY, L. H. Family - school - peer relationships: Significance for social, emotional, and academic learning. In: ZINS, J. E.; WEISSBERG, R. P.; WANG, M. C.; WALBERG, H. J. (Eds.). Building academic success on social and emotional learning. New York: Teachers College Press, 2004. p. 59-75.

COELHO, L. S. G. Encoprese e constipação em gêmeos: um estudo de caso em ludoterapia comportamental. Psicologia Ciência e Profissão, Brasília, v. 21, n. 1, p. 2-13, mar. 2001.

COUTO, M. C. V.; DUARTE, C. S.; DELGADO, P. G. G. A saúde mental infantil na Saúde Pública brasileira: situação atual e desafios. Revista Brasileira de Psiquiatria, v. 30, n. 4, p. 390-398, 2008.

CORRINGAN, P. How stigma interferes with mental health care. American Psychologist, v. 59, n. 7, p. 614-625, 2004.

COSTELlO, E. J.; FOLEY, D. L.; ANGOLD, A. 10-year research update review: The epidemiology of child and adolescent psychiatric disorders: II. Developmental epidemiology. Journal of American of Child and Adolescent Psychiatry, v. 45, p. 8-25, 2006. 
DAZZANI, M. V. M.; CUNHA, E. O.; LUTTIGARDS, P. M.; ZUCOLOTO, P. C. S. V.; SANTOS, G. L. Queixa escolar: uma revisão crítica da produção científica nacional. Psicologia Escolar e Educacional, v. 18, n. 3, p. 421-428, 2014.

DELVAN, J. S.; PORTES, J. R. M.; CUNHA, M. P.; MENEZES, M.; LEGAL, J. E. Crianças que utilizam os serviços de saúde mental: caracterização da população em uma cidade do sul do Brasil. Revista Brasileira de Crescimento e Desenvolvimento Humano, v. 20, n. 2, p. 288-237, 2010.

FU I, L.; CURATOLO, E.; FRIEDRICH, S. Transtornos afetivos. Revista Brasileira de Psiquiatria, São Paulo, v. 22, supl. 2, p. 24-27, dez. 2000.

GAUY, F. V.; ROCHA, M. M. Manifestação Clínica, Modelos de Classificação e Fatores de Risco/Proteção para Psicopatologias na Infância e Adolescência. Temas em Psicologia, v. 22, n. 4, p. 783-793, 2014.

GONÇALVES, J. A.; MOREIRA, E. A. M.; TRINDADE, E. B. S. M.; FIATES, G. M. R. Transtornos alimentares na infância e na adolescência. Revista Paulista de Pediatria, v. 31, n. 1, p. 96-103, 2013.

KENDALL, P. C.; COMER, J. S. Childhood disorders. 2. ed. London: Psychology Press, 2010.

LOPES, M. C. In/exclusão escolar: a invenção de tipos específicos de alunos. Revista Colombiana de Educación, v. 54, p. 96-119, 2008.

LOUZADA, R. C. R. Caracterização da clientela atendida no Núcleo de Psicologia Aplicada da Universidade Federal do Espírito Santo. Estudos de Psicologia, Natal, v. 8, n. 4, p. 451-457, 2003.

MELO, S. A.; PERFEITO, H. C. C. S. Características de crianças da clínica de Psicologia de 2000 a 2002. Estudos de Psicologia, Campinas, v. 23, p. 3, p. 239-249, 2006.

PATEL, V.; FLISHER, A. J.; HETRICK, S.; MCGORRY, P. Mental health of young people: A global public-health. Lancet, v. 369, p. 1302-1313, 2007.

ROCHA, M. M.; RESCORLA, L. A.; EMERICH, D. R.; SILVARES, E. F. M.; BORSA, J. C.; ARAÚJO, L. G. S.; BERTOLLA, M. H. S. M.; OLIVEIRA, M. H. S. M.; PEREZ, N. C. S.; FREITAS, P. M.; ASSIS, S. G. Behavioural/emotional problems in Brazilian children: findings from parents' reports on the Child Behavior Checklist. Epidemiology and Psychiatric Sciences, p. 1-10, 2013.

ROHDE, L. A.; BARBOSA, G.; TRAMONTINA, S.; POLANCZYK, G. Transtorno de déficit de atenção/hiperatividade. Revista Brasileira de Psiquiatria, São Paulo, v. 22, supl. 2, p. 07-11, dez. 2000.

SILVARES, E. F. M. Clínicas-escola: novas formas de atendimento psicológico. Tese (Livre-docência) - Universidade de São Paulo, São Paulo, 1998. 
TANAKA, O. Y.; LAURIDSEN-RIBEIRO, E. Ações de saúde mental na atenção básica: caminho para ampliação da integralidade da atenção. Ciências: Saúde Coletiva, v. 14, n. 2, p. 477-86, 2009.

TRESCO, K. E.; LEFLER, E. K.; POWER, T. J. Psychosocial Interventions to Improve the School Performance of Students with Attention-Deficit/Hyperactivity Disorder. Mind Brain, v. 1, n. 2, p. 69-74, 2010.

VAN ACKER, R. Antisocial, aggressive, and violent behavior in children and adolescents within alternative education settings: Prevention and intervention. Preventing School Failure, v. 51, n. 2, p. 5-12, 2007.

WEBSTER-STRATTON, C.; REID, M. J. The Incredible years parent, teachers, children training series: A multifaceted treatment approach for young children with conduct problems. In: KAZDIN, A. E.; WEISZ, J. R. (Eds.). Evidence-based psychotherapies for children and adolescents. New York: Guilford Press, 2003. p. 224-240.

WEISZ, J. R.; HAWLEY, K. M. Developmental factor in the treatment of adolescents. Journal of Consulting and Clinical Psychology, v. 70, p. 21-43, 2002.

WELSH, M.; PARKE, R. D.; WIDAMAN, K.; O’NEIL, R. Linkages between children's social and academic competence: A longitudinal analysis. Journal of School Psychology, v. 39, p. 463-481, 2001.

WEISZ, J. R.; SANDLER, I. N.; DURLAK, J. A.; ANTON, B. S. Promoting and protecting youth mental health through evidence-based prevention and treatment. American Psychologist, v. 60, n. 6, p. 628-648, 2005.

ZINS, J. E.; BLOODWORTH, M. R.; WEISSBERG, R. P.; WALBERG, H. J. The scientific base linking social and emotional learning to school success. In: ZINS, J. E.; WEISSBERG, R. P.; WANG, M. C.; WALBERG, H. J. (Eds.). Building academic success on social and emotional learning: What does the research say? New York: Teachers College Press, 2004. p. 3-22.

Texto recebido em 13 de janeiro de 2016. Texto aprovado em 13 de janeiro de 2016. 
